\title{
Kontra KBV-Konzept
}

\section{Geriatrie ist Hausarzt-Sache!}

\author{
Senioren sollen von aufwändig weitergebildeten Ärzten in Schwerpunkt- \\ praxen versorgt werden. Hält die KBV die Hausärzte für inkompetent?
}

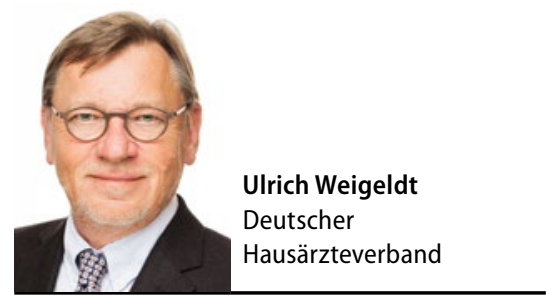

_ Man ist es ja gewohnt, dass sich Versorgungskonzepte der Kassenärztlichen Bundesvereinigung (KBV) bisweilen wie direkte Angriffe auf die hausärztliche Tätigkeit lesen. Und dennoch ist man immer wieder aufs Neue erstaunt, wie unverblümt die Selbstverwaltung von Zeit zu Zeit versucht, den Hausarztberuf auszuhöhlen. Das neuste Beispiel hierfür hat die KBV erst vor wenigen Monaten bei ihrer Herbsttagung geliefert.

In ihrem Konzept zur „spezifischen geriatrischen Versorgung“ schlägt sie vor, dass zukünftig sogenannte geriatrische Schwerpunktpraxen verstärkt in die Versorgung unserer älteren Patienten eingebunden werden sollen. Eine in der Regel 18-monatige Zusatzweiterbildung Geriatrie, die zumeist ganztägig und hauptberuflich in einer stationären Einrichtung geleistet werden muss, soll für das nötige Know-how sorgen. Es scheint offensichtlich, dass kaum ein Hausarzt seine Praxis für so lange Zeit verlassen kann, um in einer stationären Spezialeinrichtung „weitergebildet“ zu werden.

\section{Zerstörte Strukturen}

Das KBV-Konzept würde in der Praxis bedeuten, dass die Patienten aus ihren vertrauten Strukturen herausgerissen und durch die neu entstandenen Schnittstellen unnötig belastet werden. Dabei versorgt bereits der Hausarzt als der zentrale Ansprech-

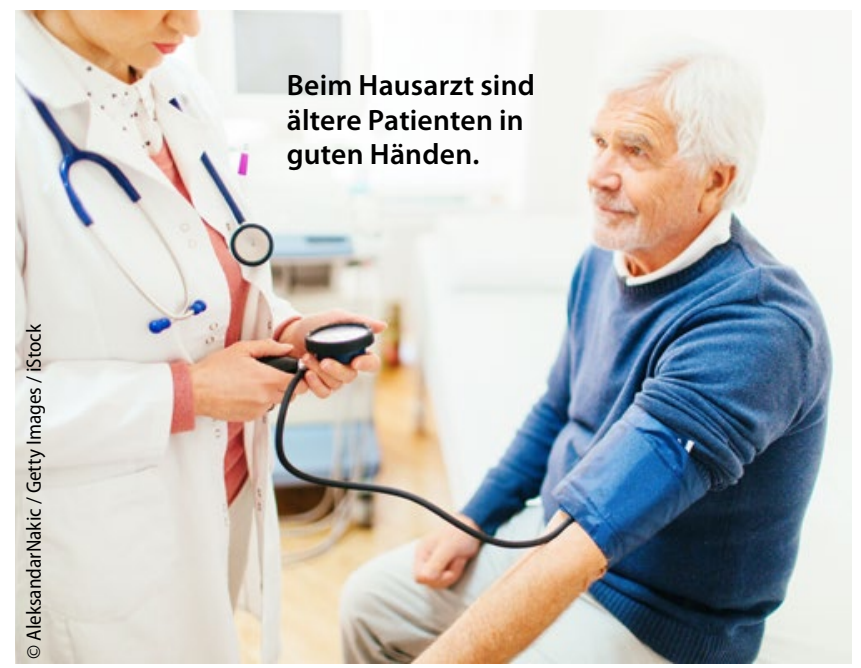

z.B. mit Hausbesuchen - dafür, dass sie möglichst lange zuhause betreut werden auch die Einbeziehung der Angehörigen.

Das alles hat der Hausazt in seiner Weiterbildung gelernt, und es ist Bestandteil seiner täglichen Arbeit in der Praxis. Dabei koordiniert er bei Bedarf die Mitbehandlung durch andere Fachärzte, Apotheker, Vertreter anderer Heilberufe und notfalls auch durch Krankenhäuser. „Schwerpunktpraxen“ als zusätzliche Schnittstellen würden diese Koordina-

Wenn durch das Geriatrie-Konzept der KBV also die Versorgung der Patienten in keinster Weise verbessert wird, können. Dazu gehört selbstverständlich tion eher erschweren als erleichtern.

arzt für Geriatrie einzuführen - es geht hier also weniger um Versorgungsfragen als vielmehr um Klientelpolitik.

Gerade dieser Spezialisierungsdrang hat zur Folge, dass es in Deutschland über 80 verschiedene Facharztrichtungen gibt - was $\mathrm{zu}$ teilweise chaotischen und unkoordinierten Versorgungsabläufen im KVSystem führt. Dass dabei en passant unser abwechs lungsreicher und vielseitiger Beruf in seinen Kompetenzen nach und nach ausgehöhlt wird, scheint in der ärztlichen Selbstverwaltung niemanden $\mathrm{zu}$ stören.

Gegen diese Diskreditierung der hausärztlichen Arbeit setzt der Deutsche Hausärzteverband seit vielen Jahren die Hausarztzentrierte Versorgung (HZV) um. In ihr wird der Hausarzt wieder ins Zentrum der Versorgung gerückt. Vermeidbare Schnittstellen und ihre Risiken werden minimiert. Da hier der Hausarzt immer erster Ansprechpartner seiner Patienten ist, sorgt er für eine bessere Koordination der Behandlungsabläufe und eine höhere Versorgungsqualität. Dies wurde inzwischen in mehreren wissenschaftlichen Evaluationen in Baden-Württemberg nachgewiesen. Diesen Weg werden wir auch mit Blick auf partner seine geriatrischen Patienten umfassend. Er kennt sie meist seit Jahren, hat ihren Alterungsprozess und ihre individuellen Ziele im Blick und sorgt - warum dann dieser Vorschlag? Der Gedanke dahinter liegt auf der Hand: Mithilfe eines scheinbaren Bedarfs wird versucht, durch die Hintertür einen Fach- das neue Geriatrie-Konzept konsequent weiterverfolgen.

- Der Autor ist Bundesvorsitzender des Deutschen Hausärzteverbands 